Meta

Journal des traducteurs

Translators' Journal

\title{
En garde !
}

\section{Jean Marcotte}

Volume 20, numéro 2, juin 1975

URI : https://id.erudit.org/iderudit/003567ar

DOI : https://doi.org/10.7202/003567ar

Aller au sommaire du numéro

Éditeur(s)

Les Presses de l'Université de Montréal

ISSN

0026-0452 (imprimé)

1492-1421 (numérique)

Découvrir la revue

Citer cet article

Marcotte, J. (1975). En garde ! Meta, 20(2), 141-150.

https://doi.org/10.7202/003567ar

Ce document est protégé par la loi sur le droit d'auteur. L'utilisation des services d'Érudit (y compris la reproduction) est assujettie à sa politique d'utilisation que vous pouvez consulter en ligne.

https://apropos.erudit.org/fr/usagers/politique-dutilisation/ 


\section{DROBLIEMES ET SOLUTIONS}

\section{EN GARDE !}

L'escrime est un des rares domaines, tant sportif que général, où le français est la langue de création et l'anglais celle de traduction. Nous allons donc aborder ce sport unique, à l'origine de l'ancien d'Artagnan comme du Zorro moderne, en nous restreignant à l'offensive et à la défensive au fleuret. Deux livres anglais ${ }^{1}$, deux livres français ${ }^{2}$ et la pratique de ce sport durant trois ans ont servi de base à cette exploration terminologique. Nous parlerons d'abord des règles régissant cette arme, puis du mouvement des pieds, des bras et de la main, des attaques, des préparations d'attaque, des parades et de la phrase d'armes.

L'escrimeur (fencer) doit protéger une certaine surface quand il tire contre un adversaire: il est alors tireur (fencer). Au fleuret (foil) la surface valable (valid target area or valid target) se limite au tronc, postérieur exclus. Une touche (hit or touch) est donc non valable (off target or invalid) au masque, et le contraire (valid) si elle touche (to strike or hit) le bas-ventre. Un coup plaqué (slap) ne compte pas. Pour éviter d'en faire, le tireur respectera sa mesure (fencing distance, fencing measure, distance or measure), c'est-à-dire la distance maximale à laquelle il peut atteindre son adversaire en se fendant. Il devra gagner la mesure pour se mettre à portée de l'adversaire, ou la rompre pour se trouver hors de portée. Les mouvements de jambes exécutés dans ce but lui permettront de placer son attaque s'il a le sens de l'à-propos (timing). Il ne reste à son adversaire qu'à fermer ou couvrir (to close, cover, or protect) la surface menacée. Il se servira pour cela de la partie près de la coquille, le fort (forte), ct non de l'extrémité de son fleuret, le faible (foible).

L'escrime est synonyme de rapidité : le tircur doit donc avoir un bon jeu de jambes (footwork), tout comme le boxcur. Il peut exécuter une marche (advance or forward step) en avançant d'abord le pied avant (leading foot) ou le pied arrière (trailing foot); il peut aussi faire une retraite (retreat or step

1. Muriel Bower et Torao Mori, Fencing, Dubuque, Iowa Brown, Physical Education Activities Series, 1972, $2^{e}$ éd., 62 p. ; Maxwell R. Garret et Mary F. Heinecke, Fencing, Boston, Allyn and Bacon, Basic Concepts of Physical Activity, 1971, 107 p.

2. Institut national des sports, Secrétariat d'Etat à la jeunesse et aux sports, Traité d'escrime le fleuret, Paris, 1963, 85 p.; Pierre Thirioux, Escrime moderne altx trois armes, Paris, Editions Amphora, 1970, 399 p. 
back) de l'un ou l'autre pied. Tous ces mouvements se font à partir de la position fondamentale (basic stance) : la garde ou position de garde (on-guard, on-guard position, or guard position) (fig. 1). Le bras armé (leading or weapon arm) est fléchi et le bras non armé (trailing arm) s'arrondit en arc de cercle, le coude à la hauteur de l'épaule. Pour toucher l'adversaire, le tireur se développera (to lunge) en utilisant la fente (lunge) (fig. 2). Le déploiement du bras suivi de la fente, qui est à proprement parler le seul mouvement des jambes, constitue le développement ${ }^{3}$. Une fois en fente (ou développement), le tireur doit reprendre la garde (to recover) pour parer la réaction adverse : il exécute une reprise de garde (return or recovery to the guard position, or recovery). Autre forme de déplacement : la flèche (flèche). Dans ce cas, le déploiement du bras est suivi d'un déséquilibre volontaire où le tireur semble courir vers son adversaire. Enfin, un saut suivi d'une fente, déplacement fulgurant comme la flèche, s'il est bien exécuté, porte le nom de Balestra (Ballestra).

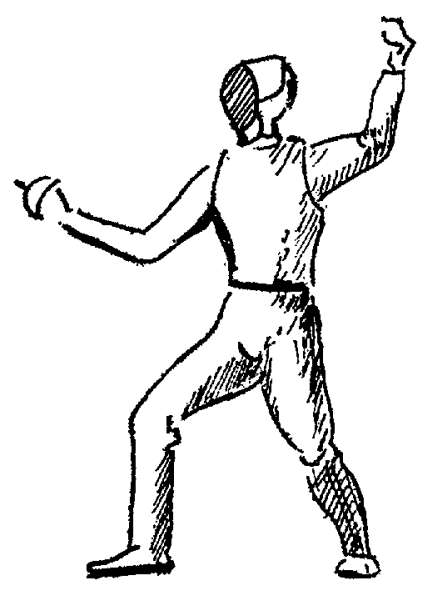

FIGURE 1

La garde

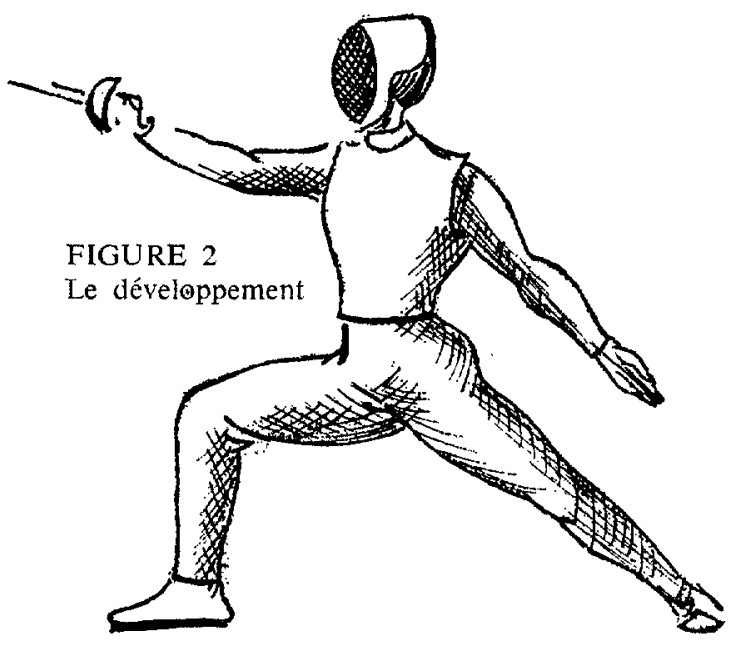

La surface valable (fig. 3) peut se diviser horizontalement en deux zones : la ligne haute (high line) et basse (low line), et verticalement en deux zones: le dehors (outside line), du côté avant du corps, et le dedans (inside line), du côté arrière du corps ${ }^{4}$. La main armée peut être en pronation, la paume au-dessus de la poignée, ou en supination, la paume au-dessous, dans chacune des quatre lignes.

3. La pratique courante substitue les expressions "fente " et « se fendre " aux termes savants " développement " et " se développer ".

4. Des maîtres d'armes spécialisent ce vocabulaire et gardent l'expression * ligne dessus " pour la ligne haute du dehors, «ligne dehors " pour la ligne basse du dehors, "ligne dessous " pour la ligne basse du dedans et a ligne dedans" pour la ligne haute du dedans (fig. 4). L'anglais ne semble pas le faire et qualifie les lignes de outside low, outside high, etc. 


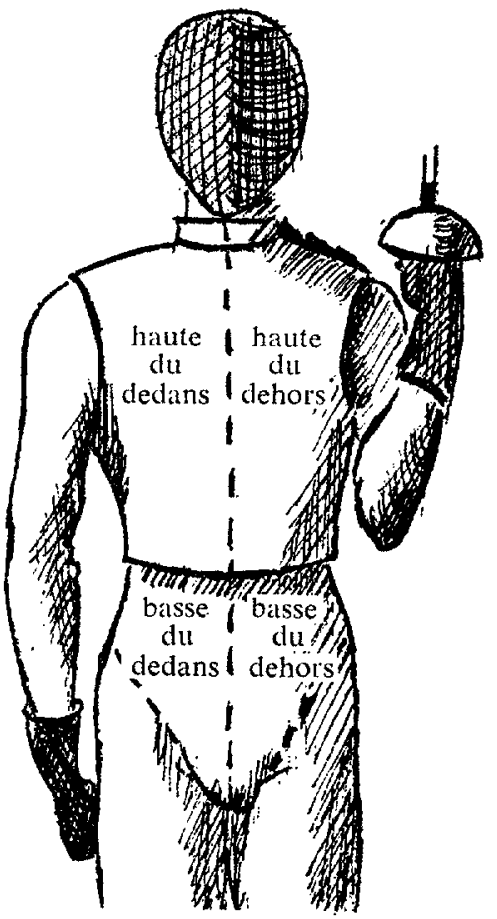

FIGURE 3a

Lignes d'un gaucher

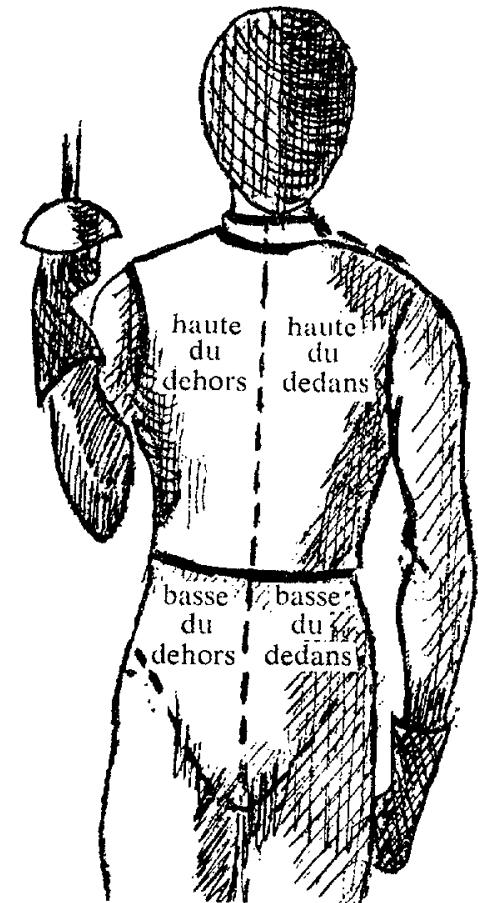

FIGURE 3b

Lignes d'un droitier

FIGURE 4

Lignes d'un droitier

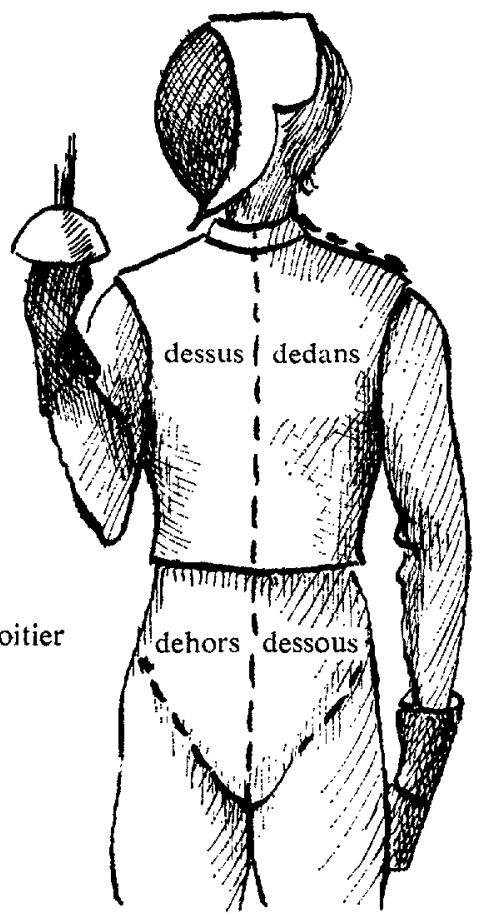


Il y a donc huit positions d'escrime (guard position), qui sont des positions de main et non de jambes ou de corps : la prime (prime), la seconde (seconde), la tierce (tierce), la quarte (quarte), la quinte (quinte), la sixte (sixte), la septime (septime) et l'octave (octave). Ces positions se prennent par rapport à ses propres lignes et non celles de l'adversaire. Si le tireur possède un bon doigté (fingerplay), c'est-à-dire la faculté de conduire et de déplacer la pointe de l'arme par la seule action des doigts, ses chances de succès sont d'autant plus grandes.

L'attaque peut se faire sans contact des fers: il y a alors absence de fer (absence of blades). Le tireur peut aussi joindre le fer adverse ou prendre l'engagement (to engage) avant d'attaquer. Une fois l'engagement pris (engaged), le tireur peut tactiquement exécuter un changement d'engagement (change of engagement) en passant dans la ligne opposée à celle dans laquelle il se trouvait. L'attaque est simple (simple) s'il n'y a qu'un mouvement, composée (compound or composed attack) dans le cas contraire. L'attaque simple est directe (direct) si elle ne change pas de ligne et indirecte (indirect) dans les autres cas. Attention! Une attaque directe peut être précédée d'un changement d'engagement, puisque ce dernier se fait avec le bras armé fléchi et que l'attaque ne débute qu'avec le déploiement du bras. Il n'y a qu'une attaque simple directe : le coup droit (straight thrust). D'une ligne, en position de garde, on déploie le bras dans cette ligne et on se fend. Il y a deux attaques simples indirectes : le dégagement (disengagement) et le coupé (coupé cutover or cut-over). Le dégagement change de ligne en passant par le chemin le plus court, le coupé en passant par devant la pointe adverse (fig. 5 et 6 ). L'attaque composée est constituée d'une ou de plusieurs feintes d'attaque (feint) suivies d'un coup final. La feinte n'est qu'un déploiement du bras et vise le même but qu'à la boxe. Une feinte en ligne basse entraîne une réaction adverse vers la ligne basse et libère la ligne haute pour une autre feinte ou le coup final, qui sera un coup droit, un dégagement ou un coupé. Chaque fois que le tireur évite la parade adverse dans son attaque composée, il trompe (to deceive a parry or evade a blade) cette parade. Certaines attaques composées portent un nom spécial. Une feinte de dégagement suivie d'un dégagement trompant une parade directe ou semi-circulaire est un une-deux (one-two). Si le dégagement qui suit la même feinte trompe une parade circulaire, l'attaque s'appelle doublement ou doublé (doublé). Si la feinte est faite en ligne dessus, par coupé ou dégagement, et la finale en ligne dessous, par coupé ou dégagement, c'est un dessus-dessous (high-low attack). Dernière méthode pour l'attaque composée: la fausse attaque (false attack) suivie du coup final. Cette fausse attaque ressemble à la feinte, sert au même but, mais se fait en fente partielle. Elle est en quelque sorte entre la feinte et le développement.

Malgré l'éventail d'attaques possibles, il est avantageux de s'assurer d'abord du fer adverse par des préparations d'attaque (preparatory action). Ce peut être des attaques au fer (attack on the blade), qui sont des actions violentes exercées sur le fer, ou des prises de fer (prise-de-fer), qui cherchent plutôt à le maîtriser. 
PROBLÈMES ET SOLUTIONS

145
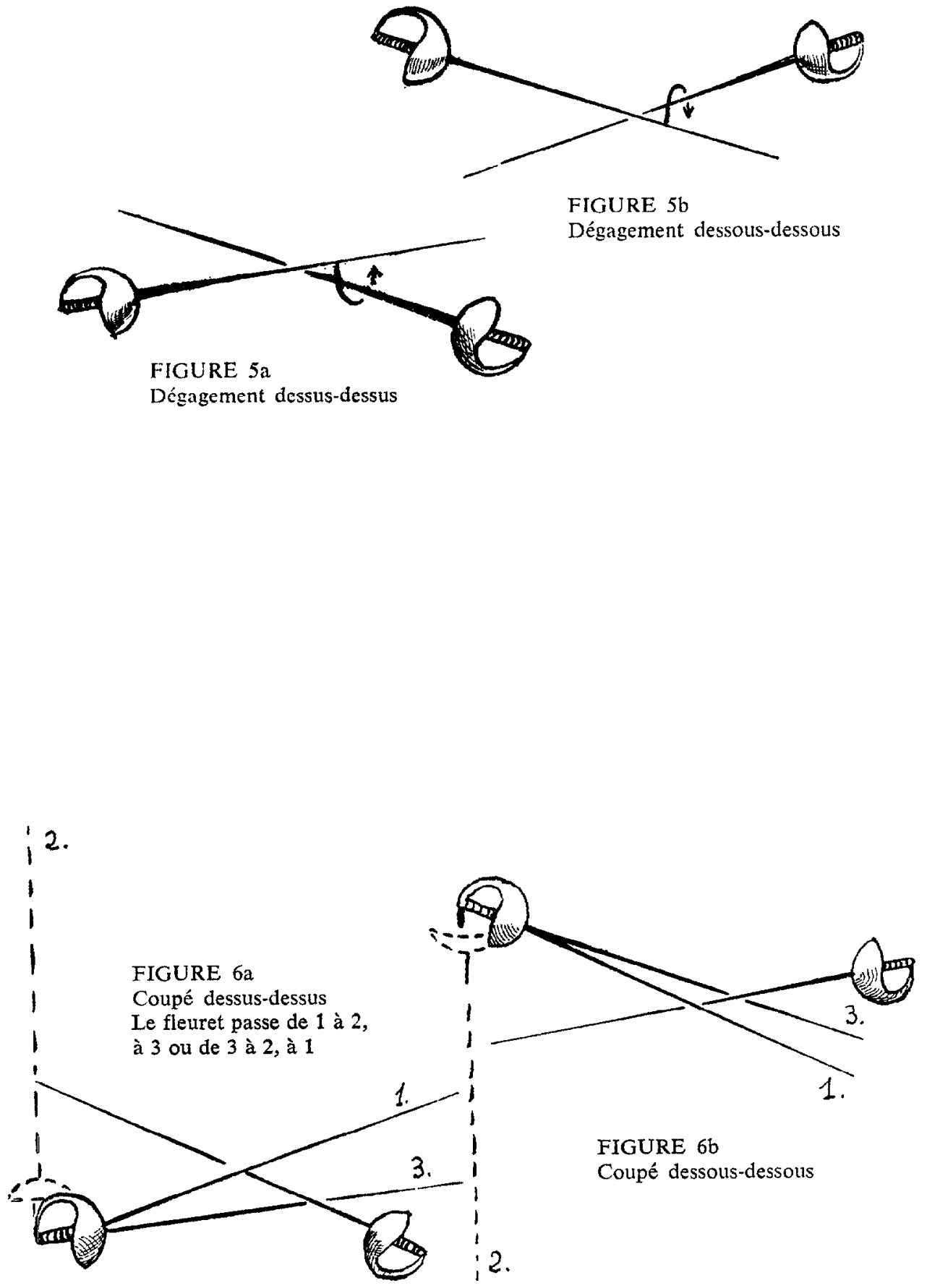
Les attaques au fer sont le battement (beat), le froissement (froissement) et la pression (pressure). Le battement est un choc exécuté latéralement sur le fer adverse pour l'écarter et ouvrir la ligne. Le tireur exécute un froissement comme la maîtresse de maison affile un couteau. La pression est une poussée exécutée sur le fer adverse après que l'engagement a été pris : c'est en quelque sorte un battement doux mais sec. Les prises de fer sont l'opposition (opposition), le coulé (glide or coulé), le croisé (croisé), le liement (bind or liément) et l'enveloppement (envelopment). L'opposition prend le fer adverse dans une ligne, le maîtrise en l'écartant, tout en le laissant dans la même ligne jusqu'à la finale de l'action. Le coulé est l'action de glisser le long du fer adverse en déployant le bras pour préparer ou loger l'attaque. Il force la lame adverse et est intimement lié à l'attaque, contrairement à l'opposition, qui se fait sur une lame souple et qui écarte d'abord le fer adverse avant l'attaque. Le croisé prend le fer adverse dans une ligne haute, l'amène dans la ligne basse correspondante (ou inversement) en le maîtrisant et en l'écartant en finale de l'action, alors que le liement fait la même chose, mais dans la ligne basse opposée (ou inversement). L'enveloppement est un mouvement circulaire autour de la lame qui entraîne et maîtrise la lame adverse tout en ramenant l'engagement dans la même ligne.

La parade (parry), e'est l'action de se garantir d'une action offensive en détournant avec son arme le fer adverse. Il y a trois sortes de parade: latérale ou directe (lateral or direct), semi-circulaire (semicircular) ${ }^{5}$ et circulaire (circular). Cette dernière porte aussi le nom de contre (counter parry or counter). La parade latérale ou directe écarte le fer dans la ligne où il se présente sans passer de ligne haute en ligne basse ou vice-versa. La parade semi-circulaire fait ce passage avant d'écarter le fer adverse. La parade circulaire contourne le fer adverse sans l'entraîner (contrairement à l'enveloppement) avant de l'écarter. Le contre s'appellera contre de sixte (counter-six) s'il se termine en sixte, contre de quarte (counter-four) s'il se ternine en quarte, et ainsi de suite. Enfin, chacune de ces parades peut être d'opposition (blocking parry) ou du tac (beat-parry). La première consiste à s'emparer du fer sans brusquerie en l'accompagnant pour l'écarter complètement de la cible; la seconde détourne le fer adverse à l'aide d'un battement sec.

Venons-en à la compétition. Une phrase d'armes (phrase or phrase d'armes) est la période qui va du «Allez»du président, l'arbitre du combat, à la première touche faite. Il y a d'abord l'attaque de l'attaquant et la parade de l'attaqué. La riposte (riposte) est le coup qui est porté par l'attaqué après avoir paré l'attaque. La contre-riposte (counterriposte) est le coup qui est porté après avoir paré une riposte ou une contre-riposte. Il y a donc l'attaque, une parade, la riposte, une parade, la première contre-riposte, une parade, la deuxième contre-riposte, etc. Rares sont les phrases d'armes aussi parfaites, car plusieurs « anomalies » surgissent en cours de combat. Si l'attaqué ne pare pas et attaque lui aussi, il exécute une contre-attaque (counterattack ${ }^{6}$ ). Alors que la contre-riposte suit la riposte, la contre-attaque et l'attaque sont simultanées. Si cette contre-attaque ne prend pas

5. Des maîtres font la même différence entre parade semi-circulaire et parade diagonale qu'entre croisé et liement.

6. Counterattack prend parfois le sens de "riposte » en anglais. 
le fer adverse, c'est un arrêt (stop) ; si elle le prend, c'est un coup de temps (time thrust). L'attaque est prioritaire à moins que la contre-attaque ne réussisse à prendre un temps d'escrime (fencing time), notion vague et difficilement perceptible dans un combat. La définition que l'on en donne est celle de la durée d'exécution d'une action d'escrime simple. Supposons $A$ qui attaque par une-deux et $B$ qui contre-attaque par coup droit. Les deux tireurs se touchent en même temps. Si $B$ a contre-attaqué avant le début du mouvement final de $A$, il a gagné un temps d'escrime et a la priorité de touche. Une autre anomalie est une riposte qui ne vient pas immédiatement après la parade, une riposte à temps perdu (delayed riposte). Ce genre de riposte peut inciter l'attaquant à remiser (to remise), à faire un redoublement (redoublement) ou une reprise d'attaque (reprise). La remise (remise) est un replacement de la pointe après une parade adverse, et ce, sans fléchir le bras armé ni reprendre la garde. La Fédération internationale d'escrime (F.I.E.) définit le redoublement comme un replacement de la pointe après une parade adverse non suivie de riposte. Contrairement à la remise, il y a repli du bras, mais comme dans la remise, il n'y a pas de reprise de garde. La reprise d'attaque est un replacement de la pointe après une parade adverse, et ce, après une reprise de garde ${ }^{7}$. Si l'attaque est précédée d'une préparation comme le battement, l'attaqué peut soustraire son fer à cette préparation et devancer ainsi l'attaque d'un temps d'escrime. Il exécute de la sorte un dérobement (derobement or deception) : il dérobe son fer au fer adverse. Le dérobement et le trompement des attaques composées s'exécutent de la même façon. Le premier évite un mouvement de l'attaquant; le second, un mouvement de l'attaqué. Enfin, si la préparation est une pression, l'attaqué peut faire une contre-pression (counter-pressure), ou pression en réponse à la première, qui lui donne une priorité d'attaque.

J'espère que cet exposé profitera aux traducteurs du domaine'sportif. Il est évident que la traduction vers l'anglais y trouvera davantage de ressources, le français étant la langue de création. Cependant, l'anglais crée déjà un vocabulaire sans équivalents français et les différents auteurs, tant français qu'anglais, présentent souvent des conceptions et des terminologies divergentes. Cette exploration très technique se veut avant tout un phare pour les traducteurs vers les deux langues. Puisse-t-elle leur permettre de ramener les particularités d'un auteur aux chemins communs du vocabulaire de l'escrime.

JeAn Marcotte

7. Quelques maîtres intervertissent les définitions de redoublement et de reprise d'attaque. Comme les compétitions internationales suivent les règles de la F.I.E., il est préférable d'adopter les définitions de la Fédération. 


\section{VOCABULAIRE FRANÇAIS-ANGLAIS}

absence de fer à-propos

arrêt

arrière, adj.

attaque au fer

avant, adj.

Balestra

battement

bras armé

bras non armé

changement d'engagement

composée (attaque, riposte, etc.)

contre, $\mathrm{n}$.

contre-attaque

contre de quarte

contre de sixte

contre-pression

contre-riposte

coulé, $\mathrm{n}$.

coup d'arrêt

coup de temps

coup droit

coupé, $\mathrm{n}$.

coup plaqué

couvrir

croisé

dedans, $\mathrm{n}$.

dégagement

dehors, $n$.

dérobement

dessus-dessous

développer, se

directe (attaque, riposte, etc.)

(voir aussi : parade directe)

doigté

doublé, $\mathrm{n}$.

doublement

engagement (voir atssi : prendre

l'engagement)

enveloppement

escrimeur

faible, $\mathrm{n}$.

fausse attaque

feinte d'attaque, feinte

fente

fermer

flèche

fleuret

fort, $n$.

froissement

garde (voir aussi : reprendre la garde et reprise de garde)

indirecte (attaque, riposte, etc.) absence of blades

timing

stop, $\mathrm{n}$.

trailing, adj.

attack on the blade

leading, adj.

Ballestra

beat, $\mathrm{n}$.

leading arm, weapon arm

trailing arm

change of engagement

composed, compound (attack, risposte, etc.)

circular parry, counter parry, counter

counterattack $\left(\mathrm{S} / \mathrm{G}^{8}\right)$

counter-four

counter-six

counter pressure

counter-riposte

glide, coulć

cf. arrêt

time thrust

straight thrust

coupé, cut-over, cutover, $\mathrm{n}$.

slap, $\mathbf{n}$.

to cover, to protect, to close

croisé

inside line

disengagement

outside line

deception, derobement

high-low attack

to lunge

direct (attack, riposte, counter-riposte)

fingerplay

doublé, $n$.

doublé, $\mathbf{n}$.

engagement

envelopment

fencer

foible, $\mathbf{n}$.

false attack

feint, $\mathrm{n}$.

lunge, $n$.

to close, to protect, to cover

fleche

foil, $\mathbf{n}$.

forte, $n$.

foissement

on-guard, on-guard position ; guard position indirect (attack, riposte, counter-riposte)

8. $S / G$ exprime un rapport de générique à spécifique entre le terme français et anglais. 
jeu de jambes

liement

ligne

ligne basse

ligne haute

marche

mesure

non valable

octave

opposition (voir : parade d'opposition)

parade circulaire

parade directe

parade d'opposition

parade du tac

parade latérale

parade semi-circulaire

phrase d'armes

plaqué

position de garde

position d'escrime

position fondamentale

prendre l'engagement

préparation d'attaque

pression

prime

prise de fer

quarte (voir : contre le quarte)

quinte

redoublement

remise, $\mathrm{n}$.

remiser

reprendre la garde

reprise d'attaque

reprise de garde

retraite

riposte à temps perdu

seconde, $\mathrm{n}$.

septime

simple (attaque, riposte, etc.)

sixte (voir aussi : contre de sixte)

surface non valable

surface valable

temps d'escrime

temps perdu, à

tierce

tireur

touche non valable

touche valable

toucher, v.

tromper une parade

une-deux

valable footwork

bind, liément

line

low line

high line

advance, forward step

fencing, distance, fencing measure, measure, distance

cf. surface non valable et touche non valable octave

opposition

circular parry

lateral parry, direct parry

blocking parry

beat-parry

lateral parry, direct parry

semicircular parry

phrase, phrase d'armes

cf. coup plaqué

on-guard, on-guard position ; guard position

guard position $(S / G)$

basic stance

to engage

preparatory action

pressure

prime

prise-de-fer

quarte

quinte

redoublement

remise, $\mathrm{n}$.

to remise

to recover

reprise

return to the guard position, recovery to

the guard position, recovery

retreat, step back, $\mathrm{n}$.

delayed riposte

seconde, $n$.

septime

simple (attack, riposte, counter-riposte)

sixte

invalid target area, invalid target

valid target area, valid target

fencing time

cf. riposte à temps perdu

tierce

fencer $(S / G)$

invalid hit, invalid touch, off target hit, off target touch

valid hit, valid touch

to hit, to strike

evade the opponent's blade $(S / G)$;

deceive a parry

one-two

cf. surface valable et touche valable 